\title{
Implementación de un objeto de aprendizaje: el caso de una docente de enseñanza secundaria
}

\author{
Implementation of a learning object: the case study of a secondary \\ education teacher
}

\section{*María Camila Castillo Cabezas \& **Alfonso Claret Zambrano}

Castillo, M. C., \& Zambrano, A. C. (2020). Implementación de un objeto de aprendizaje: el caso de una docente de enseñanza secundaria. Revista Convergencia Educativa, 8, diciembre, 6-30. DOI: http://doi.org/10.29035/rce.8.6

[Recibido: 25 agosto, 2020 / Aceptado: 19 diciembre, 2020]

\section{RESUMEN}

El presente trabajo de investigación analiza los resultados del proceso de implementación de un Objeto de Aprendizaje (OA) de Ciencias Naturales por parte de una docente en servicio con más de 20 años de experiencia en la enseñanza secundaria. Se justifica si se considera que al transcurrir la última década un porcentaje importante de las investigaciones sobre OA han puesto el foco en asuntos de orden técnico, mientras que las implicaciones y promesas educacionales permanecen no probadas empíricamente en particular en el aula de la escuela secundaria. Ahora bien, la metodología se efectúa desde la perspectiva cualitativa descriptiva por estudio de casos. Siguiendo las siguientes fases: fase de contextualización; fase de inmersión y fase de resultados y análisis de la implementación del OA. Las fuentes de datos incluyeron observaciones en el aula, grabaciones de audio y video, entrevistas semiestructuradas y planes de área y aula, los datos fueron examinados mediante el análisis en profundidad. Los resultados permitieron establecer que los $0 A$ son vinculados a una propuesta curricular concreta de formación docente en la institución educativa, donde el currículo prescrito por el OA dialoga con el currículo planificado y desarrollado por el docente, en este diálogo bidireccional el flujo de aprendizaje propuesto sufre modificaciones para apoyar el aprendizaje y la enseñanza.

Palabras clave: TIC, Objeto de Aprendizaje, enseñanza de las Ciencias Naturales, docente en servicio.

\footnotetext{
* Universidad del Valle, Santiago de Cali, Colombia. ORCID ID: https://orcid.org/0000-0003-4086-9686

** Universidad del Valle, Santiago de Cali, Colombia. ORCID ID: https://orcid.org/0000-0003-4795-6037
} 
Castillo, M. C., \& Zambrano, A. C. (2020). Implementación de un objeto de aprendizaje: el caso de una docente de enseñanza secundaria. Revista Convergencia Educativa 8, diciembre, 6-30. DOI: http://doi.org/10.29035/rce.8.6

\section{ABSTRACT}

This research paper analyses the results of the implementation process of a Natural Science Learning Object (LO) by an in-service teacher with more than 20 years of experience in secondary education. It is justified considering that over the last decade a significant percentage of research on LO has focused on technical issues, while the educational implications and promises remain unproven, particularly in the secondary school classroom. The methodology carried out in this research is qualitative descriptive by means of a case study, through the following phases: contextualization phase; immersion phase; and results and analysis phase of the implementation of the LO. The data sources included classroom observations, audio and video recordings, semi-structured interviews, and area and classroom plans. The data were examined through in-depth analysis. The results allowed establishing that the LOs are linked to a concrete curricular proposal for teacher training in the educational institution, where the curriculum prescribed by the LO dialogues with the curriculum planned and developed by the teacher, in this two-way dialogue the proposed flow of learning undergoes modifications to support learning and teaching.

Key words: ICT, Learning Object, Natural Science teaching, in-service teacher.

\section{Introducción}

En los últimos años, una de las propuestas que se están manejando en el ámbito Internacional y Nacional del aprendizaje basado en tecnología, es la organización de contenido educativo en la forma de objetos de aprendizaje (en adelante OA) como un recurso digital que apoya el aprendizaje de los estudiantes (Wiley, 2002). A nivel nacional una de las reformas del currículo estatal formulas con el firme propósito de mejorar la calidad de la educación básica, primaria y secundaria fue el diseño y desarrollo de OA. Se trata de recursos tecnológicos que entraron a formar parte del plan de fortalecimiento curricular como uno de los referentes para la planeación curricular con los que docentes en las áreas de Matemáticas, Ciencias Naturales y Lenguaje cuentan para desarrollar la enseñanza-aprendizaje-evaluación. Los mismos se encuentran alojados en forma de cápsulas educativas en el portal de colombiaprende, (www.colombiaaprende.edu.co), desde donde puede ser descargado, granulado y reorganizado para el acto educativo.

Conviene mencionar, que el marco teórico utilizado como referente para la producción de los OA fue el modelo ADDIE acrónimo del proceso de Análisis, diseño, desarrollo, implementación y evaluación, cinco etapas que en la práctica no fueron totalmente desarrolladas por el Centro de Innovación Educativa Regional Zona Sur (CIER-SUR), ya que fueron omitidas las fases de implementación y evaluación.

Al respecto, en el manual de producción y gestión de contenidos educativos digitales para profesionales del Ministerio de Educación Nacional, 2014 propone avaluar la actualización conceptual del OA, aspectos pedagógicos como los objetivos, metas de aprendizajes y presupuestos para la evaluación formativa, aspectos eminentemente educativos, pero que en ningún caso reemplazan la evaluación in situ del recurso como lo propone el modelo. 
Castillo, M. C., \& Zambrano, A. C. (2020). Implementación de un objeto de aprendizaje: el caso de una docente de enseñanza secundaria. Revista Convergencia Educativa 8, diciembre, 6-30. DOI: http://doi.org/10.29035/rce.8.6

En consecuencia, la presunción que los OA apoyan el aprendizaje de los estudiantes con la cual se justificó el masivo diseño y desarrollo de estos recursos a nivel nacional, no ha sido probado empíricamente, en consecuencia hasta la fecha existe muy poca información acerca de cómo estos recursos son integrados al proceso de enseñanza-aprendizaje-evolución en la secundaria (Kay \& Knaack 2007, Kay \& Knaack, 2009, Collazos, Pantoja, Hernández, Solarte, Agredo \& Vásquez, 2007, González, Gómez \& Abrego, 2014, González, 2016).

Los OA son recursos de apoyo al aprendizaje, por lo que una baja calidad en los recursos y/o la carencia de evaluaciones sistemáticas afecta al proceso de aprendizaje. Lo anterior, hace necesario la evaluación del producto mismo (evaluación durante el desarrollo del OA) como de la implementación en contextos reales (la evaluación del uso). En otras palabras, no basta sólo con analizar las actividades de aprendizaje del OA, sino que se requiere obtener una retroalimentación de los usuarios finales que los utilizan en los contextos cotidianos. De ahí que, el presente trabajo de investigación analiza los resultados del proceso de implementación de un Objeto de Aprendizaje (OA) de Ciencias Naturales por parte de una docente en servicio con más de 20 años de experiencia en la enseñanza secundaria.

\section{Las políticas TIC en los sistemas educativos: caso Colombia}

Asistimos a un mundo efímero, globalizado, lleno de subjetividades que compiten a diario con la ciencia y la tecnología por enculturizar y dominar al hombre. En esta lucha incesante, las tecnologías de la información y la comunicación (TIC) están encausando nuevas formar y medios de relacionamiento socioafectivo, nuevas formas de entender, actuar y convivir con el otro y lo otro, el mundo cada vez más automatizado está transformando todas las esferas a nivel sociocultural. Como resultado, las TIC están haciendo cada vez más presencia en la escuela, motivando consigo la formulación de políticas públicas que buscan en la integración de las TIC la ruta para la innovación y calidad educativa. Al respecto, las Naciones Unidas (2013), a través de los objetivos de desarrollo del milenio, se convirtió en una de las primeras políticas en abogar por la integración de las TIC a nivel educativo, específicamente cuestiones como el acceso a las nuevas tecnologías deberían ser solventadas en colaboración con el sector público y privado de las naciones.

Atendiendo a los objetivos del milenio, en la última década las líneas de prioridad de las políticas educativas gubernamentales de los países de América Latina y el Caribe estuvieron centradas en formalizar la transición hacia la sociedad de la información, implementando proyectos, y estrategias principalmente vinculadas al tema de infraestructura de las telecomunicaciones, equipamiento y conectividad.

En el caso colombiano específicamente, son varias las políticas transversales de TIC que se han promulgado desde los años 90 hasta hoy. En este sentido, la tecnología e informática aparece como un "área obligatoria y fundamental de la educación básica”. En la Ley general de educación (Ley No. 115, 1994), se 
Castillo, M. C., \& Zambrano, A. C. (2020). Implementación de un objeto de aprendizaje: el caso de una docente de enseñanza secundaria. Revista Convergencia Educativa 8, diciembre, 6-30. DOI: http://doi.org/10.29035/rce.8.6

establece su incorporación al currículo por medio de la Resolución №2343 de 1996, reforzando la idea de "entrar" a la sociedad global (de la información y del conocimiento). Además, el artículo $\mathrm{N}^{\circ} 32$, en referencia a la educación media técnica, establece la necesidad imperante de formar a los estudiantes tanto en asuntos técnicos como científicos, facilitando su relacionamiento y adaptación al mundo de la vida (Congreso de Colombia, 1994).

Posteriormente, en el Plan Nacional de Desarrollo 1998-2002 se establece como prioridad el acceso universal a los servicios de telecomunicaciones. Fue ejecutado en los años 1999-2001, a través del Programa Compartel de Telefonía Social, con el propósito de instalar puntos de telecomunicaciones comunitarios en la totalidad de los municipios del país. Hoy el Plan Vive Digital 2014-2018, se propone consolidar a Colombia como país líder en el desarrollo de aplicaciones con utilidad social para promover el progreso de los colombianos más pobres; ser el Gobierno más eficiente y transparente con apoyo en la tecnología y promover y fortalecer la formación de talento digital y llenar la autopista de la información con servicios, aplicaciones y contenidos que permitan solucionar los problemas de los ciudadanos, consolidando así un país en paz, más equitativo y más "educado".

Ahora bien, estas políticas que promueven la integración de las TIC en la educación se pueden analizar a la luz de los programas y proyectos desarrollados en las instituciones educativas oficiales del País, presentado varios énfasis. A saber:

(i) Equipamiento, caso Computadores para Educar y proyecto Tit@. El primero, creado en el 2002 para ayudar a cerrar la brecha digital con iniciativas que responden a políticas multisectoriales, a finales de 2013 reporta haber dotado 780.000 terminales (entre computadores y tabletas) y capacitado a 313.000 docentes en 40.000 sedes. El segundo, en alianza con el Ministerio de Tecnología y Comunicaciones y la Alcaldía de Cali, invirtió $\$ 30.000$ millones para beneficiar a 34.104 estudiantes y 2463 docentes.

(ii) Formación continua de docentes y directivos docentes, se implementaron iniciativas como el proyecto Tit@, programas de formación e investigación del CIER-SUR, Intel, Computadores para educar y ciudadano digital; estos programas se ubican en el marco del Plan Nacional de Desarrollo 2006 - 2016 que propone transformar la práctica pedagógica de docentes y directivos a través de la integración y uso apropiado de las TIC al aula (Galvis, López \& Aarón, 2018).

(iii) Producción y gestión de contenidos educativos; una de las iniciativas más actuales de este énfasis es la creación de los Centros de Innovación Educativa Regional (CIER), encargados de la capacitación de docentes y programas de investigación. Asimismo, desde el año 2004 se implementa a nivel nacional el portal Colombia Aprende (www.colombiaaprende.edu.co), donde se encuentran alojados en forma de cápsulas educativas una gran variedad de contenidos educativos 
digitales para ser consultados por toda a la comunidad educativa, especialmente para las áreas de matemáticas, ciencias naturales y lenguaje. Conviene destacar que las investigaciones sobre cómo este tipo de recursos educativos son puestos en práctica en los contextos reales de aula son limitadas, por lo que este trabajo realiza un importante aporte al campo, explicitando la toma de decisiones curriculares y de enseñanza de un docente al implementar este tipo de recurso.

\subsection{A qué se refiere el término OA y sus principales características}

Una de las más grandes dificultades en la investigación de los $\mathrm{OA}$ se debe a la falta de consenso respecto a su significado, situación que problematiza su diferenciación respecto a recursos tecnológicos de otra naturaleza. Así, autores como García (2005), Astudillo (2011), Callejas (2011), Chiappe (2009), Polsani (2003) y Wiley (2002) han definido los objetos de aprendizaje de forma indistinta, sin llegar a un consenso tácito. Esta falta de acuerdo es una de las evidencias del estado de desarrollo inicial en el cual se encuentra esta nueva perspectiva educativa. No obstante, como afirman Wiley (2002) y Balatsoukas, Morris \& Brien (2008) "la proliferación de definiciones para el término 'objeto de aprendizaje' hace la comunicación confusa y difícil".

Pese a la falta de consenso, los autores mencionados, coincidieron en incluir dentro de sus definiciones una serie de rasgos distintivos, a saber: (i) Recursos digitales; (ii) Independientes; (iii) Reutilizables y (iv) Que apoyan el aprendizaje. Ahora bien, en el año 2006, el Ministerio de Educación Colombiano (MENC) y por ende los Centros de Innovación Educativa Regional (CIER) basándose en la definición de Cisco Systems define un OA como:

"conjunto de recursos digitales, autocontenible y reutilizable, con un propósito educativo y constituido por al menos tres componentes internos: contenidos, actividades de aprendizaje y elementos de contextualización. Con una estructura de información externa (metadatos) que facilite su almacenamiento, identificación y recuperación" (Astudillo, 2011, p. 20, MEN, 2011, p. 16).

Además, las características particulares que presentan los OA planteados para un currículo colombiano son: (i) Material del profesor: donde se encuentra toda la propuesta curricular base para el desarrollo del $\mathrm{OA}$ en el aula de clases. (ii) Interfaz: donde se encuentran las diferentes actividades para su desarrollo y (iii) Material del estudiante: donde se encuentra la propuesta curricular. Así pues, al transcurrir la última década un porcentaje importante de las investigaciones sobre OA han puesto el foco de atención en asuntos de orden técnico, mientras que las implicaciones y promesas educacionales permanecen no probadas empíricamente particularmente en el aula de la escuela secundaria. Lo anterior llama la atención acerca de la necesidad de investigar como los OA son integrados al proceso de enseñanza-aprendizaje y evaluación de las ciencias (Polsani, 2003, Balatsoukas, Morris \& Brien, 2008, Hsu, 2017). 
Resaltamos que la pregunta de investigación que se resuelve de manera teórica y empírica es: ¿cómo una docente en servicio con más de 20 años de experiencia en la enseñanza secundaria implementa un Objeto de Aprendizaje (OA) de Ciencias Naturales?; se trata de un cuestionamiento relevante si se considera que El Ministerio de Educación Nacional Colombiano ha apuntado hacia el desarrollo de habilidades tecnológicas en el manejo de las TIC y no en la formación docente, desconociendo con esto la complejidad del acto educativo y la relevancia del contexto.

\section{Aspectos metodológicos}

Este estudio empleó un "diseño de estudio cualitativo básico " de perspectiva descriptiva por estudio de casos. Las fuentes de datos incluyeron observaciones en el aula, grabaciones de audio y video, plan de área y aula y entrevistas semiestructuradas. El desarrollo metodológico siguió las siguientes fases:

Fase de contextualización. Caracterización de la población de esta investigación; los participantes fueron una docente de enseñanza secundaria y su grupo de estudiantes. Por confidencialidad su seudónimo es Diana, trabajaba en una institución educativa oficial, cuenta con 20 años de experiencia en la enseñanza de las Ciencias Naturales y una amplia formación en TIC, pues ha participado en varios de los programas de cualificación docente ofrecidos por el estado (Intel introductorio, Ciudadano digital, Tit@ y Entre pares).

Se trata de 28 estudiantes de noveno grado de una institución educativa oficial de la ciudad de Santiago de Cali, cursan la jornada de la tarde, matriculados en el año escolar 2018, asistentes al curso de Ciencias Naturales (Biología), cuyas edades comprenden entre los 14 y 15 años, pertenecen a un sector de la población en alto riesgo físico, moral y social, y, en su mayoría, a una estratificación socioeconómica baja.

Cabe destacar que, este estudio se llevó a cabo en la sede central dotada con recursos tecnológicos como sala Tit@, tabletas, computadores portátiles y conectividad entre otros. En otras palabras, esta sede de la institución educativa cumple con las condiciones básicas para llevar a cabo la implementación de un objeto de aprendizaje.

Fase de inmersión: Durante esta fase se llevaron a cabo tres entrevistas semiestructuradas para comprender los razonamientos y acciones inteligentes de la docente: (a) entrevista de antecedentes, (b) entrevista previa observación y (c) entrevista posterior a la observación. La docente tuvo la oportunidad de volver a revisar la lección y articular las razones de sus decisiones curriculares y de enseñanza.

Hay que mencionar, además, que se realizó una observación no participante de las clases de la docente Diana, abordando el tema de Herencia Biológica en uno de los cinco grupos que tenía a su cargo, el cual estaba integrado por 28 estudiantes. Para abordar el tema, la docente dedicó ocho sesiones de 50 minutos cada una. 
Respetando la autonomía de la docente respecto a la planificación y desarrollo del acto educativo, se le permitió tomar todas las decisiones curriculares y de enseñanza que considerara necesarias. Dichas decisiones se presentan en distintos momentos; antes, durante y después de la clase y van desde la revisión del plan de área y aula para determinar metas de aprendizaje, la implementación de los elementos del OA en clase, hasta la verificación de aprendizajes. Así la primera decisión fue seleccionar el OA ¿Cómo se expresa la información genética? S_G08_U02_L08 para el grado 9.

Fase resultados y análisis de la implementación del OA. El desarrollo de esta fase de la investigación implicó analizar los datos mediante el análisis en profundidad. En este enfoque, primero se identificó un segmento (episodio) específico de enseñanza, grabado en video o durante las entrevistas, posteriormente se realizó una descripción detallada del episodio en términos de: (a) razonamientos y acciones inteligentes del profesor, el lenguaje y las formas en que interactúa con los estudiantes, (b) acciones de los estudiantes, (c) patrones comunes entre el currículo prescrito por el $\mathrm{OA}$ y el desarrollado por la docente.

\section{Resultados y análisis}

Responder la pregunta de investigación que se plantea, no solo implica identificar los razonamientos y acciones inteligentes del profesor, el lenguaje y las formas en que interactúa con los estudiantes, sino que, entender cómo está configurado este recurso tecnológico, otorga sentido y significancia a dichas acciones. Por lo tanto, en las siguientes líneas se realiza un análisis preliminar del OA "cómo se expresa la información genética" desde el punto de vista pedagógico. Posteriormente, se presentan los resultados del proceso de implementación agrupados en tres grandes categorías de análisis sustentadas cada una por episodios identificados tanto en el proceso de observación no participante o captados durante la entrevista semiestructurada, a saber: (i) transformación del flujo de aprendizaje sugerido por el OA: en busca de la identidad profesional; (ii) dificultades técnicas, de conexión e infraestructura y (iii) interés y la motivación.

\subsection{Análisis preliminar del $\mathrm{OA}$; "cómo se expresa la información genética" desde el punto de vista pedagógico}

El análisis documental, tanto del instrumento manuscrito, como de la interfaz y el material del estudiante que estructuran el OA, presenta una orientación de enseñanza centrada en el profesor y el contenido, y en menor medida focalizada en el estudiante, lo que evidencia que su diseño está fundamentado en las teorías del aprendizaje conductista y procesamiento de la información.

Esta afirmación se puede visualizar en los recursos digitales y tareas problemas utilizados para representar los contenidos y procesos de pensamiento de esta disciplina, constituidos en una alta proporción en imágenes fijas, videos, animaciones, actividades interactivas (juegos de relación, juegos de arrastrar y soltar), en su conjunto, direccionados a predecir hechos. Esta situación lleva a inferir que el 
diseñador concibe la enseñanza como el proceso por medio del cual se comunican conocimientos a fin de cambiar conductas a través de la administración y supervisión de ambientes de aprendizaje.

Algo semejante ocurre con las actividades que configuran este ambiente de aprendizaje; un número reducido de estas propende por la discusión, reflexión, formulación de hipótesis y análisis de situaciones problemas, lo cual promueve la comunicación, negociación, toma de decisiones y resolución de problemas en los estudiantes. Simultáneamente, el OA presenta actividades de aprendizaje que son una fuente transmisora de conocimientos de naturaleza factual. En otras palabras, estas actividades se limitan a representar y formular en un formato digital, los diferentes elementos teóricos que configuran un contenido particular, con miras a que los estudiantes logren memorizar la conceptualización de un fenómeno natural (conductismo), o, en el mejor de los casos, relacione la nueva información con el conocimiento que yace en sus memorias permanentes (procesamiento de la información).

Ahora bien, el análisis documental realizado a la estructura lógica del manuscrito deja ver claramente que los diseñadores de éste descuidaron incluir las dificultades/concepciones alternativas con las que llega el estudiante al aprendizaje, características contextuales, potencialidades que ofrecen los recursos de naturaleza analógica y digital para el aprendizaje de un contenido, recursos analógicos o digitales para gestionar el aula, pedagogía general (técnicas, estrategias y modelos de enseñanza), y estrategias de evaluación. Como consecuencia de la ausencia de los elementos curriculares antes mencionados al OA, probablemente le hace falta una concordancia intracurricular que articule coherentemente los diferentes elementos de la enseñanza.

La situación anterior puede llegar a generar una ruptura entre los OA y la propuesta estatal del Plan de Integración de los Componentes Curriculares (por ejemplo, lineamientos curriculares, EBC, DBA, matriz de referencia). De hecho, los OA no son recomendados de manera clara como un recurso curricular que ayuda a mediar el aprendizaje en los estudiantes, con el mismo nivel de importancia que los otros componentes curriculares prescriptos por las políticas públicas.

\subsection{Transformación del flujo de aprendizaje sugerido por el OA: en busca de la identidad profesional}

El análisis a los planes de área y aula, así como la observación no participante del proceso de implementación del OA “cómo se expresa la información genética" realizado por la docente Diana, indicaron que la misma no asume el $\mathrm{OA}$ como una propuesta curricular inmutable, sino que la transforma de acuerdo a sus necesidades. En otras palabras, varias de las actividades implementadas por la docente a lo largo del proceso de Enseñanza-aprendizaje-evaluación no fueron conjeturadas por el OA, sino que son producto del proceso de reflexión sobre la acción, del reconocimiento de las limitaciones/dificultades de los estudiantes y las características del contexto educativo. 
Castillo, M. C., \& Zambrano, A. C. (2020). Implementación de un objeto de aprendizaje: el caso de una docente de enseñanza secundaria. Revista Convergencia Educativa 8, diciembre, 6-30. DOI: http://doi.org/10.29035/rce.8.6

El episodio (E1) muestra cómo la docente Diana transforma el flujo de aprendizaje sugerido por el OA adicionando una actividad que ella ha implementado en años anteriores, y que sabe, ha dado resultado a la hora de enseñar el tópico de genética, además, le otorga identidad profesional. Así, sobreponiendo su conocimiento sobre lo que los estudiantes han aprendido en años anteriores y la forma y/o estrategias que le han dado resultado, decide hacer uso de una situación problema alterna a la propuesta en el OA, llamada El caso de Sam Berns: Progeria.

Esta situación problema plantea el caso de Sam Berns, un joven de diecisiete años de edad que sufre de progeria, mutación genética no hereditaria que causa el envejecimiento brusco y prematuro en niños; a través de esta situación problema, la docente aplica su conocimiento sobre el currículo horizontal ya que con la misma, busca generar el contexto propicio para articular el OA con los temas abordados durante el periodo escolar, además de otorgarle una base o sustento en un caso de la vida real, situación que es también acordada por el área de Ciencias Naturales de la institución educativa donde labora.

Lo anterior es explicitado en la siguiente declaración:

(Entrevista semiestructurada: $\mathrm{P}$ = profesor, $\mathrm{I}$ = investigador)

I: ¿Realizó alguna modificación al flujo de aprendizaje?

P: En la medida en que se iba desarrollando las OA se iban realizando algunas modificaciones, uno decía, no para la próxima si le tengo que cambiar esto, porque esto con estos niños no me va a ir bien.

Me pasaba mucho con las lecturas, conociendo al grupo yo sabía que esas lecturas estaban un poco complejas para el grupo, mire que incluso el $O A$ que utilizamos era para el grado octavo, pero para ese grupo esa lectura era muy elevada y yo sabía que iba a seguir teniendo dificultades con ellas, entonces les busqué una lectura que fuera más accesible para ellos, más entendible, más fácil, entonces por eso cambiábamos, para facilitarle la comprensión a ellos.

I: Usted decide hacer uso de una situación problema alterna a la que sugiere el OA, ¿Qué la motivó a realizar esta adicción a la propuesta curricular sugerida por el OA?

P: Sí señora, yo quise poner un ejemplo que estuviera más cerca de la vida de los estudiantes, sería chévere conectar esa parte tan científica con ellos, algo que los toque, que haga la temática más cercana, porque las lecturas tenían su complejidad y yo entiendo que tengan este nivel, porque la ciencia no anda en chancletas, pero al menos alguna actividad como que se relacionara más con ellos en la cotidianidad. P: Que entiendan que la genética también tiene que ver con ellos, que ellos también tienen ADN, por eso utilicé los de Sam Berns, porque con él ellos se dan cuenta de lo que pasa internamente; mire las 
Castillo, M. C., \& Zambrano, A. C. (2020). Implementación de un objeto de aprendizaje: el caso de una docente de enseñanza secundaria.

Revista Convergencia Educativa 8, diciembre, 6-30. DOI: http://doi.org/10.29035/rce.8.6

consecuencias que puede tener, entonces sí, las lecturas científicas de rigor hay que darlas, pero faltan otras que aterricen las lecturas a donde estamos, para que los muchachos vean que eso también tiene que ver con ellos.

Además, trabajar con situaciones problemas es algo que se plantea como área.

Otro episodio (E2) que vale la pena mencionar, es la diferencia que existe entre los objetivos del OA y el fin último que persigue la docente para sus estudiantes. Como se mencionó líneas arriba, algunas actividades se limitan a representar y formular en un formato digital los diferentes elementos teóricos que configuran un contenido particular, con miras a que los estudiantes logren memorizar la conceptualización de un fenómeno natural. Contrario a esto, la docente Diana no solo busca que los estudiantes aprendan Ciencias, sino también, desarrollar las habilidades lingüísticas como la oralidad, la lectura y la escritura comprensiva, lo que a escalas más altas no solo apoya la colaboración y desarrollo de la oralidad y la escucha, sino también los llevaría a desarrollar procesos de pensamiento superior.

Lo anterior es explicitado en la siguiente declaración:

(Entrevista semiestructurada: $\mathrm{P}$ = profesor, $\mathrm{I}$ = investigador)

I: ¿Por qué decide apostarle al desarrollo de habilidades lingüísticas (oralidad, lectura y escritura)?

P: Yo creo que el proceso queda más completo si primero lo tratan de interiorizar por ellos mismos y luego lo hacemos grupal y en voz alta [...] Primero lo vamos leyendo, utilizando la vista, luego lo volvemos a ver y lo escuchamos y luego contestamos preguntas, entonces yo creo que ahí está como más o menos completo el proceso, esto les ayudaría mucho porque el Icfes es sobre todo comprensión de texto.

P: yo siempre estoy apuntando a que ellos desarrollen los procesos de pensamiento superior, el análisis, la reflexión y la síntesis, eso es lo que yo quiero. Entonces, cuando ellos desarrollan eso, va a ser más fácil asociar el ADN con el gen, con el cromosoma, ellos van desarrollando estos procesos de pensamiento superior que en este grado es a lo que uno apunta.

Así pues, aprovechando que el OA descuidó en su diseño aspectos de la pedagogía general como técnicas de gestión de aula y estrategias de enseñanza-evaluación, decide hacer uso de su conocimiento pedagógico para implementar estrategias como lecturas individuales y grupales de textos cortos con un contexto sociocultural ubicando la idea principal y secundaria, así como la construcción de textos de determinado género que estén dirigidos a una audiencia particular con un propósito científico.

La docente hace uso de la estrategia de lectura guiada donde el cuerpo del texto es leído de manera pausada por toda la clase, es decir, la idea representada en cada uno de los párrafos al igual que su microestructura se constituye en el centro de discusión de los estudiantes. Posteriormente, cada estudiante 
Castillo, M. C., \& Zambrano, A. C. (2020). Implementación de un objeto de aprendizaje: el caso de una docente de enseñanza secundaria. Revista Convergencia Educativa 8, diciembre, 6-30. DOI: http://doi.org/10.29035/rce.8.6

debería realizar una lectura individual del texto para afinar de manera personal aquellos aspectos faltantes por comprender. Esta estrategia apoya el desarrollo del lenguaje formal de las ciencias.

Por otro lado, pese a que las animaciones y los videos ayudan a visualizar las entidades y procesos de las ciencias que son extremadamente pequeños, grandes, rápidos y lentos, éstos en un alto porcentaje, tienden a estimular una orientación de enseñanza centrada en el contenido o el profesor, ya que comunican unidireccionalmente una información que representa un contenido. Por el contrario, la docente Diana utiliza una forma distinta de gestionar el video en el aula, identifica incidentes críticos del mismo, y los vuelve objeto de reflexión a través de cuestionamientos dirigidos a los estudiantes, lo cual abre espacios de diálogos e interacciones estudiante-video, estudiante-estudiante y estudiante-docente que no estaban considerados en la linealidad del OA.

Para saldar esta categoría de manera puntual me referiré al episodio (E3), que pone en escena la preponderancia de actividades que abogan por el desarrollo de aspectos de orden conceptual, dejando de lado lo procedimental, actitudinal, lo sociocultural y la creatividad como referente de formación para los estudiantes. Se trata de dos actividades no conjeturadas por el OA, pero que la docente Diana, valiéndose de su sistema de conocimientos, valores y creencias, decide adicionar, ya que responden no sólo a aspectos de orden actitudinal y procedimental, sino también rompen con la idea de ciencia como verdad absoluta e inalterable, busca que sus estudiantes comprendan que la ciencia es ante todo una actividad cultural que tiene implícitamente un sentido creativo.

Así pues, la mayoría de las actividades de aprendizaje del OA de ciencias, tuvieron como interés central el representar y formular un conjunto de productos de las ciencias (por ejemplo, Contenidos/Habilidades). Sin embargo, descuidaron la representación y formulación de los procesos por medio de los cuales se construyen los modelos teóricos. Es decir, en este OA no se está representando de manera explícita la naturaleza de las ciencias, que es práctica, creativa, ampliamente política y definitivamente cultural.

La primera actividad que se adiciona a la propuesta curricular sugerida por el OA corresponde a la construcción de un modelo a escala del ADN, donde los estudiantes deberían utilizar sus conocimientos sobre la estructura del ADN, su creatividad, ingenio e imaginación para construir este modelo a escala; no existieron orientaciones sobre la forma de construcción, los materiales o recursos a utilizar, la única instrucción fue el tiempo de entrega estimado.

Vale la pena destacar que este tipo de actividades, donde los estudiantes ponen el juego todos sus sentidos, se comunican, se apoyan en sus compañeros, trabajan en equipo y crean, son altamente asertivas para la construcción de aprendizajes significativos, que posteriormente pondrán en juego en otros contextos y escenarios, no solo educativos, sino también sociales. Además, la construcción de un modelo a 
Castillo, M. C., \& Zambrano, A. C. (2020). Implementación de un objeto de aprendizaje: el caso de una docente de enseñanza secundaria. Revista Convergencia Educativa 8, diciembre, 6-30. DOI: http://doi.org/10.29035/rce.8.6

escala del ácido desoxirribonucleico (ADN) puede mediar el establecimiento de relaciones conscientes entre los niveles concretos y abstractos del mundo biológico.

Lo anterior es explicitado en la siguiente declaración:

(Entrevista semiestructurada: $\mathrm{P}=$ profesor, $\mathrm{I}=$ investigador)

P.: ... algunos contenidos eran bastante complejos y netamente científicos, noté que a pesar de todo lo que se les dijo, lo que vimos en los videos, las preguntas que se hicieron, las imágenes que se trabajaron, ellos todavía no parecían entenderlo. Entonces decidí cambiar de estrategia y hacerlo manual, a ver si de una forma más concreta y creativa lo entendían un poco más, en verdad no podían entenderlo entonces era para hacerlo más concreto para ellos.

La segunda actividad hace referencia a un debate sobre los alimentos transgénicos, en esta ocasión la docente decide utilizar un video seleccionado por ella misma y que ha hecho parte de su repertorio de recursos y actividades por muchos años. El mismo, tiene el propósito de informar a los estudiantes acerca de las características e implicaciones sociales y económicas alrededor de los organismos genéticamente modificados (OGM), lo cual les ayudará a que adquieran una postura crítica en torno al panorama científico, político, económico y cultural alrededor de este tema.

Así, la docente Diana utiliza la estrategia del trabajo en equipo para que los estudiantes realicen una investigación que les permita construir los argumentos necesarios para defender su postura alrededor de los OGM; cada integrante del grupo asumió un rol dentro del mismo (coordinador, investigador, cohesionador y presentador o defensor de la posición). Pasados 20 minutos abre el debate otorgando la posibilidad a los estudiantes, no solo de que expongan y defiendan sus ideas, sino también de reconocer otros puntos de vista, los comparen con los propios y modifiquen lo que piensan ante argumentos más sólidos.

El empleo de esta estrategia de Enseñanza-Aprendizaje-Evaluación contribuyó a que los estudiantes desarrollaran competencias que mejoran considerablemente su aprendizaje, como es el cultivo de la retórica y de la argumentación a partir de la contraposición de ideas y argumentos. Lo anterior, no solo mejora las habilidades de comunicación y expresión oral, sino que también, desarrollan su capacidad investigadora a partir de un trabajo de consulta y de preparación de las intervenciones.

Más aún, el empleo de esta estrategia permitió que los estudiantes adquieran una postura crítica y reflexiva en torno al panorama científico, político, económico y cultural alrededor de los alimentos transgénicos, que a futuro permitirá la toma de decisiones informadas a nivel personal, local, nacional y global que mejoren la sociedad, además de contribuir a superar la visión de "neutralidad" y "objetividad" que tradicionalmente se ha atribuido a la ciencia. Por una visión donde el hombre es un ser social que determina y es determinado por su contexto. 
Castillo, M. C., \& Zambrano, A. C. (2020). Implementación de un objeto de aprendizaje: el caso de una docente de enseñanza secundaria.

Revista Convergencia Educativa 8, diciembre, 6-30. DOI: http://doi.org/10.29035/rce.8.6

Lo anterior es explicitado en la siguiente declaración:

(Entrevista semiestructurada: $\mathrm{P}$ = profesor, $\mathrm{I}$ = investigador)

I: ¿Por qué decide agregar esta actividad en la secuencia de enseñanza-aprendizaje-evaluación?

P: Porque la consideré pertinente, ya que estamos hablando de genoma, de leyes de la herencia, de ingeniería genética; es que la ciencia no es solo leyes y teorías, detrás de ella hay todo un contexto político que el OA no desarrolla. Por ejemplo, en lo de Watson y Crick, el OA no menciona que ellos se basaron en el trabajo de Rosalind Franklin y eso tiene que ver con el papel de la mujer en la ciencia. Por esto, también puse lo de los transgénicos, porque ese tema no responde solo a lo científico, sino que el tinte social y político que tiene lo vuelve muy controversial.

En suma, como lo plantean Coll, Onrubia \& Mauri (2007) y Coll (2008), cada grupo de participantes (profesores y estudiantes) redefine y recrea los procedimientos y normas "teóricas" para la enseñanzaaprendizaje-evaluación, haciendo uso de herramientas tecnológicas, a partir de una serie de factores: conocimientos previos, expectativas, motivación, contexto institucional y socio institucional, entre otros.

Lo anterior es claro, toda vez que nos permite comprender por qué, a pesar de que varias de las actividades realizadas por la docente Diana no fueron conjeturadas por el OA durante su diseño, fueron puestas en escena por ella durante la implementación de este. Así por ejemplo, a pesar de que el OA no tiene en cuenta los aspectos de orden procedimental, actitudinal, lo sociocultural y la creatividad como referente de formación, pues se da prioridad a los contenidos temáticos, la docente, desde su sistema de conocimiento, valores y creencias, toma la decisión de transformar el flujo de aprendizaje sugerido por el OA e incluir actividades que sí respondieron a los aspectos antes mencionados, con el fin de representar el tópico en consideración y apoyar el aprendizaje de los estudiantes.

Así pues, la organización de la enseñanza particular de la docente Diana jugó un papel importante, pues logró transformar el sentido de las actividades que estaban originalmente centradas en el docente y el contenido, hacia unas donde el estudiante fuera el protagonista de su propio aprendizaje. Por ejemplo, logró pasar de actividades con una marcada orientación de enseñanza convencional, focalizada en la memorización, recuerdo de información y clarificación de conceptos, hacia unas donde primó el desarrollo de las habilidades lingüísticas como la oralidad, lectura y escritura, y la comprensión de los tópicos de ciencias, a partir de situaciones problemas que fueran relevantes para los estudiantes, actividades donde interactuaran con sus compañeros y pudieran comunicar sus ideas.

Es claro entonces que, aunque existe una diversidad de expresiones curriculares (currículo de las Ciencias, el currículo planificado por las políticas públicas y el currículo institucional), es la práctica educativa en el aula, los conocimientos profesionales de los docentes y estudiantes los que terminan dando forma y recreando el acto educativo que es reproducido socioculturalmente. Ahora, es importante 
Castillo, M. C., \& Zambrano, A. C. (2020). Implementación de un objeto de aprendizaje: el caso de una docente de enseñanza secundaria. Revista Convergencia Educativa 8, diciembre, 6-30. DOI: http://doi.org/10.29035/rce.8.6

considerar lo siguiente: a pesar de que la Ley General de Educación o Ley № 115 de 1994, los marcos generales y lineamientos curriculares de las ciencias naturales y la educación ambiental, reconocen en cierto sentido, la autonomía intelectual de los docentes en el ejercicio profesional de la enseñanzaaprendizaje-evaluación, situándose como autor y practicante de sus propias teorías, técnicas y prácticas pedagógicas y curriculares, pareciera prevalecer aún en el imaginario de los tomadores de decisiones del contexto estatal e institucional, la imagen del docente como ejecutor de un currículo que previamente ha sido diseñado por otros; como resultado de lo anterior, algunas propuestas educativas, pese a que originalmente provienen del currículo estatal, presentan un modelo pedagógico débil y dificultades de coherencia curricular.

Más aún, considerando que el éxito del OA con respecto a los logros alcanzados por los estudiantes, depende no sólo del OA en sí mismo, sino también, del sistema de conocimientos, creencias y valores del docente, quien lo pone en escena en el aula (Blancas \& Rodríguez, 2013). Resultaría conveniente hacer partícipe a los docentes del proceso de diseño de este tipo de recursos educativos, utilizando todo el conocimiento profesional que han acumulado por años de experiencia.

La categoría que sigue a continuación se encuentra íntimamente relacionada con la anterior, ya que puede llegar a modificar las prácticas de aula haciendo uso de un $\mathrm{OA}$, no necesariamente motivado por el sistema de conocimiento, valores y creencias de quien lo implementa, sino por las características del contexto de implementación. En este caso nos referimos al contexto de una institución educativa de carácter oficial.

\subsection{Dificultades de infraestructura, equipamiento y conectividad}

Asistimos a un mundo efímero, globalizado, lleno de subjetividades que compiten a diario con la ciencia y la tecnología por enculturizar y dominar al hombre. En esta lucha incesante, las tecnologías de la información y la comunicación (TIC) están encausando nuevas formar y medios de relacionamiento socioafectivo, nuevas formas de entender, actuar y convivir con el otro y lo otro; el mundo cada vez más automatizado está transformando todas las esperas a nivel sociocultural. Como resultado, las TIC cuentan cada vez con mayor presencia en la escuela; en respuesta, se han generado políticas públicas que han venido aunando esfuerzos para adelantar procesos que faciliten el acceso y la capacitación en el uso y apropiación de las TIC por parte de la comunidad académica y administrativa de las instituciones educativas.

Así, uno de los programas más recientes fue la iniciativa con énfasis en producción y gestión de contenidos educativos de calidad. En otras palabras, los OA responden a la preocupación global por incorporar las TIC de forma efectiva al proceso de formación. No obstante, vale la pena preguntar: ¿cuentan las instituciones educativas oficiales del país con la infraestructura, conectividad y capacidad instalada, para hacer uso de los OA? Los episodios que siguen demuestran que, si bien es cierto, el estado está realizando 
millonarias inversiones en la compra de tecnología, capacitación e infraestructura, se hacen necesarios mayores esfuerzos para que la integración de las TIC, en general, y la implementación de los OA, en particular, tengan mayor incidencia en el mundo educativo de la escuela.

El episodio (E4) muestra cómo la docente Diana, en una de las aulas, tiene la intención de trabajar la actividad 1, que aborda la introducción del OA. Para esto, tiene presupuestado entregar a cada uno de los estudiantes un computador personal considerado como parte de los recursos digitales del aula de Biología. La docente seleccionó previamente a un monitor, encargado de repartir estos computadores a sus compañeros, quien informó que al revisar la unidad de carga y almacenamiento (UCA), no se encuentra disponible un computador por estudiante, por lo que la docente toma la decisión de aprovechar esta situación para motivar el trabajo en pequeños grupos y para hacer uso de otros recursos como los teléfonos celulares.

Lo anterior es explicitado en la siguiente declaración:

Videos clase

P: “Muchachos, la mayoría de los equipos que tenemos ahí (señala la UCA) tienen la licencia vencida y la pantalla aparece negra, no han venido a darnos la clave o hacer reparación, entonces para qué sacar unos equipos que no van a alcanzar ni siquiera de a dos, ¿cuál va a hacer la dinámica? Vamos a formar grupos para consultar la información que necesitan y aprovechan para socializar entre ustedes; también pueden hacer uso de sus celulares".

La situación anterior es muy recurrente en la dinámica educativa de las instituciones oficiales que han sido dotadas con equipos tecnológicos en el marco de programas como "Computadores para Educar" y "Compartel". Es común que estos tengan un tiempo de vida muy corto, posiblemente asociado a la licencia digital o la clave de producto, que en muchas ocasiones no son conocidas por los administrativos y docentes. Esta situación genera que las orientaciones de enseñanza pensadas para ser desarrollas uno a uno, cambie hacia formas colectivas de trabajo que, si bien, favorecen las formas de socializar entre los estudiantes, no siempre son la metodología apropiada para todo tipo de actividad.

Lo anterior es explicitado en la siguiente declaración:

(Entrevista semiestructurada: $\mathrm{P}=$ profesor, $\mathrm{I}=$ investigador)

I: ¿De un total de 36 computadores cuantos están disponibles para usarse?

P: Creo que la mitad, mire (empieza a contar los computadores no disponibles) son 18 los computadores que no tienen licencia.

I: ¿Cómo dificulta el trabajo con el OA el hecho que los computadores no tengan licencia, y que por tanto no se puedan utilizar uno a uno? 
P: [...] El OA sería más fácil de trabajar si todos los computadores estuvieran bien, si tuviéramos una buena conectividad a internet porque uno simplemente les sube el documento y ellos lo leerían desde sus puestos, pero pues usted ve lo que nos toca hacer, proyectarlo y leerlo desde el tablero, tenemos que poner un ritmo general porque no se puede que cada uno lo lea y lo interiorice las veces que necesite, entonces nos toca, así como a la maldita sea qué cosa tan horrible.

El gobierno luego quiere calidad y excelencia con estos estados así, uno tiene mucha voluntad y todo, pero se necesitan herramientas, miren como estamos trabajando, no hay textos, no hay laboratorios, no hay biblioteca y no funcionan los portátiles, pero si calidad, calidad y en el Icfes tienen que salir excelentemente, ellos piden, pero no dan.

Otro aspecto que mencionar es el poco seguimiento a la escalabilidad y sostenimiento de los proyectos y programas pues, en muchas ocasiones, las instrucciones educativas son dotadas de equipos, pero no se realizan jornadas de mantenimiento, actualización de licencias y reemplazo de los productos. En otras palabras, el proceso de acompañamiento a los docentes, después de la culminación de los programas, es mínimo, lo que genera que, con el pasar de los años, los equipos se vuelvan obsoletos y se reduzca su uso, lo que lleva a que la implementación del programa no sea sostenido y escalable en el tiempo.

Otra situación que se suma a la anterior tiene que ver con las formas organizativas de las instituciones educativas; la dotación tecnológica, la conectividad y la infraestructura cambia dependiendo de la sede y el aula donde se realiza el proceso de enseñanza-aprendizaje-evaluación (bibliotecas, salas de profesores, salones de clase, laboratorios y otros espacios dispuestos). En otras palabras, no todas las sedes y aulas cuentan con la misma disponibilidad de recursos, así la focalización de la dotación debe ser considerada por los docentes durante la implementación de los OA.

Lo anterior es explicitado en la siguiente declaración:

(Entrevista semiestructurada $\mathrm{P}$ = profesor, I = investigador)

P: Mi clase no siempre es en este salón.

I: ¿La clase va a depender de en qué salón te toque?

P: Exactamente, por ejemplo, si llega ahorita una compañera ve necesito proyectar este video ya había programado entonces yo le digo hágale porque yo la mayoría del tiempo tengo la sala, entonces, tampoco quiero apropiarme. Debo ser responsable de la sala con los objetos, pero tampoco puedo ser egoísta, entonces imagínese, en ciencias somos, póngale, 12 maestros en la mañana y, de esos doce, cuántos trabajamos en las salas, solamente 1, entonces, ahí hay un desbalance partiendo de eso. 
La situación anterior pone en evidencia la realidad de las instituciones educativas oficiales y la deuda que aún tiene el estado con éstas en términos de calidad estética y las condiciones de infraestructura de los ambientes educativos. Es claro que las condiciones básicas de estética educativa (luz, ruido, ventilación), mejores condiciones de infraestructura, de distribución de la capacidad instalada en todos los espacios ocupados de la institución, constituyen una condición sine qua non para generar una atmósfera óptima que promueva los procesos de enseñanza y aprendizaje, además se trata de un aspecto indispensable para garantizar el derecho a la educación.

Finalmente, una de las necesidades básicas para trabajar con los OA de manera online es una buena conectividad. El episodio (E5) ejemplifica cómo, durante la implementación de la actividad de desarrollo 1 del OA, la docente Diana se ve sorprendida por la baja velocidad de navegación del internet en la institución, mientras los estudiantes se encontraban observando un video enfocado en la genética humana, para hacer referencia a la herencia de rasgos entre las familias, éste se pausaba constantemente provocando la dispersión de la clase, por lo que la docente decide hacer uso del material del estudiante (formato pdf) donde se encuentra una lectura que relaciona el tópico en cuestión.

Así, la poca conectividad obligó a la docente Diana a trabajar con los OA de manera offline, esto implicó cambiar no solo la orientación de la enseñanza, sino también la forma de gestionar y organizar el aula para la puesta en escena de la lectura guiada a través de pequeños grupos.

Lo anterior es explicitado en la siguiente declaración:

Videos clase

\section{P: Muchachos, el internet en el colegio se puso lento, entonces vamos a dejar el video hasta ahí, más bien} vamos a leer el texto ¿Cómo se relaciona el ADN con la herencia?, que se encuentra en el material del estudiante; formen grupos para repartirles los computadores por favor y realizamos la lectura guiada.

Considerando lo anterior, se vuelve imperativo que, durante la puesta en marcha de las políticas educativas que promueven la incorporación de las TIC en la educación, se tenga más en cuenta el contexto educativo y, por ende, las condiciones reales en las cuales dichos procesos se desarrollan. Por ejemplo, las dificultades de infraestructura y conectividad presentes en muchas instituciones educativas rurales y urbanas inciden en el uso y apropiación de estos recursos, por lo que una mayor cantidad de actividades que puedan ser ejecutadas de manera offline podrían constituirse en una buena alternativa para promover un mayor uso de estos recursos.

Lo anterior es explicitado en la siguiente declaración:

(Entrevista semiestructurada: $\mathrm{P}$ = profesor, $\mathrm{I}$ = investigador)

I: ¿El propósito de trabajar con los OA, qué dificultades ha llevado? 
Castillo, M. C., \& Zambrano, A. C. (2020). Implementación de un objeto de aprendizaje: el caso de una docente de enseñanza secundaria. Revista Convergencia Educativa 8, diciembre, 6-30. DOI: http://doi.org/10.29035/rce.8.6

P: Uno, la conectividad, otro, los computadores o classmate están bloqueados, no tienen internet, están desconfigurados (teclado,) otros, no tienen acceso a la red, la carga a veces no se genera completamente. Lo que se quería es que cada estudiante tenga su computador para el trabajo entonces, dotaron con 40, pero en el grupo son 46, entonces digamos, desde lo político dicen: la educación tiene que mejorar porque los profesores tienen todas las herramientas; pero no son suficientes.

Hace un tiempo, algunos computadores se bloquearon, al parecer hay un sistema que los bloquea automáticamente porque se venció la licencia, no sé exactamente lo que sucedió, aparecía un aviso que era por la falta de uso, pero, realmente, los computadores se usan, pero se bloquean, entonces no se podían utilizar las salas, pero desde allá entonces se exige ustedes deben trabajar con eso, pero si no hay un buen funcionamiento las salas, aquí por fortuna estoy solo. Y hay muchas aulas que no están dotadas, son 35 aulas dotadas y las 65 restantes, no. Causan traumatismos, por ejemplo, yo programo mi clase, entrego los computadores y materiales, en este caso, un recorte de prensa, darles un vídeo, y llega otro profesor, y vea, necesito la sala, entonces hay que llegar a acuerdos, este caso, yo soy un poco sensible, yo veo como me defiendo y me voy a otro salón, pero hay otros profesores que no sueltan la sala.

Finalmente, la enseñanza-aprendizaje- evaluación con base en las TIC, en general, y los OA, en particular, que se asume al interior de una institución educativa no solo depende de quienes producen, transforman y enseñan, es decir, los docentes, sino también, las condiciones en las cuales dichos procesos se desarrollan, la infraestructura respectiva, la conectividad y otros factores están directamente relacionados con el éxito del diseño e implementación.

Es importante destacar que las investigaciones a nivel internacional reportan resultados similares en cuanto a la orientación pedagógica que guía la implementación de estos recursos tecnológicos. En esta línea, Natriello (2005) citado por Díaz (2006) señala que aún persiste en las aulas de ciencias una concepción tradicional, netamente memorística, cambiando la pizarra por un computador, tablet o en este caso OA.

Asimismo, Moreira (2010) afirma que convertir las TIC en un elemento complementario de la enseñanza, ocasiona que no exista por parte de los docentes un replanteamiento o cambio pedagógico estructural, curricular y procedimental del acto educativo. En este sentido, la integración de las TIC es un proceso que va más allá de la proyección de un video en clase, se trata de un cambio del pensamiento docente, adquisición de nuevos roles y formas de interactuar en el aula. En última instancia, la disponibilidad de recursos tecnológicos en el aula, no supone un cambio de inmediato en el sistema de conocimiento, valores y creencias del docente (Coll, 2008, Blancas \& Rodríguez, 2013, Moreira, 2010). 


\subsection{Interés y la motivación}

El episodio (E6) relata cómo la actividad 1.2. (interfaz dos) sugiere el empleo por parte de los estudiantes de un recurso interactivo "concéntrese con imágenes" cuyo propósito es relacionar conceptos básicos de genética (cromosoma, ADN, nucleótido, gen y proteína) con imágenes que representaban el fenómeno biológico, consiste en dar clic sobre cada carta para destaparla y armar la pareja correcta. Para su implementación en el aula, la docente Diana proyecta, con ayuda del video beam el recurso interactivo, el cual ya tiene descargado y guardado en su computador; explica brevemente en qué consiste, establece una regla simple de participación y elige al azar a un estudiante que realice la actividad.

Cabe destacar que la implementación de esta actividad interactiva generó en los estudiantes un interés inmediato, logró captar su atención, generando el deseo de ser partícipes activos, no solo de esta actividad, sino de las venideras. Se mejoró significativamente las relaciones que comparten los estudiantes, dejando de lado el egocentrismo y ensimismamiento propio de estas edades.

Más aún, el cumplimiento de reglas de juego (respetar los turnos y tiempos de participación y las opiniones de los compañeros) contribuyen al desarrollo de diversas competencias sociales de los estudiantes como la emocional, cognitiva, social, física, comunicativa, así como al aprendizaje de destrezas y pensamiento reflexivo debido a que reta al estudiante a razonary pensar para dar respuesta a situaciones concretas.

Pese a lo anterior, se debe agregar que el recurso en su diseño no plantea un adecuado proceso de comunicación y ajuste de resultados (retroalimentación) que explique o justifique detalladamente al estudiante por qué la pareja que armó no es la correcta, y qué implicaciones tiene este hecho, tampoco permite corregir errores (feedback), identificar y corregir concepciones intuitivas, no se comunica al estudiante qué debe hacer para optimizar sus resultados, esta situación no contribuye a mejorar el aprendizaje.

La docente Diana se percata rápidamente de lo anterior; gracias a su reflexión en la acción decide realizar una pequeña explicación donde puntualiza aspectos ya desarrollados en clases anteriores como la estructura y función del ADN, pero que son importantes para la comprensión de lo acontecido con la actividad interactiva.

En definitiva, la mayoría de los recursos presentes en el OA son videos, imágenes en formato JPG, animaciones y textos en formato PDF. Sería conveniente entonces una mayor cantidad de recursos interactivos en formato HTML, ya que contribuyen a que los procesos abstractos del mundo biológico sean más fáciles de asimilar; es preciso también que estos sean complementados con tareas problemas que brinden la oportunidad de identificar y desarrollar procesos de razonamiento y pensamiento superior. 
Castillo, M. C., \& Zambrano, A. C. (2020). Implementación de un objeto de aprendizaje: el caso de una docente de enseñanza secundaria. Revista Convergencia Educativa 8, diciembre, 6-30. DOI: http://doi.org/10.29035/rce.8.6

\section{Conclusiones}

El objetivo general de esta investigación fue analizar los resultados del proceso de implementación de un Objeto de Aprendizaje (OA) de Ciencias Naturales por parte de una docente en servicio con más de 20 años de experiencia en la enseñanza secundaria. Así pues, se identificó y analizó los razonamientos y acciones inteligentes del profesor, el lenguaje y las formas en que interactúa con los estudiantes; los resultados permiten concluir que la incorporación de un $\mathrm{OA}$ al proceso de Enseñanza-Aprendizaje-Evaluación está atravesado por la planificación del proceso formativo. Se trata de un proceso situado y contextualizado que incluye un análisis específico y concreto del $\mathrm{OA}$ en general, y de los contenidos, objetivos, actividades de enseñanza y aprendizaje, formas de organización, y la oferta de herramientas tecnológicas en específico, que responden a las expectativas, motivación, contexto institucional y socio institucional de cada docente y sus estudiantes.

En otras palabras, los OA son vinculados a una propuesta curricular concreta de formación docente en la institución educativa, donde el currículo prescrito por el OA dialoga con el currículo planificado y desarrollo por el docente, en este diálogo bidireccional el flujo de aprendizaje propuesto sufre modificaciones para apoyar el aprendizaje y la enseñanza.

Lo anterior, se pone en evidencia cuando la docente Diana intentando responder a aspectos de orden procedimental, actitudinal, lo sociocultural y la creatividad como referente de formación, que según ella son dejados de lado por el OA, plantea actividades (debate sobre los alimentos transgénicos, situación problema: el caso de Sam Berns, modelo a escala del ADN ) que cobran sentido para los estudiantes por representar los aspectos conceptuales a partir de situaciones de la vida real en donde dichos conceptos están en juego, al mismo tiempo contribuyen al desarrollo de desarrollo de competencias (básicas, laborales, ciudadanas), habilidades y destrezas que mejoran considerablemente el aprendizaje.

Es importante agregar, que la implementación del OA dependerá, en buena medida, de las características del contexto de implementación, la naturaleza y características del equipamiento, recursos tecnológicos, dificultades de infraestructura y conectividad presentes en muchas instituciones educativas rurales y urbanas. Es necesario que se tenga más encuentra el contexto colombiano para un futuro proceso diseño y desarrollo de contenidos educativos digitales. Otro aspecto a considerar es el diseño y producción de recurso digitales educativos coherentes con perspectivas pedagógicas contemporáneas, más centradas en los estudiantes y sus características que el contenido sintáctico, también se hace necesario el uso de más recursos interactivo como simuladores, laboratorios virtuales, herramientas que le permitan al estudiante construir sus propias animaciones, software libres para apoyar a representación y formulación una disciplina comprensiva para otros. 
Unas de las limitaciones del presente estudio es la cantidad de objetos de aprendizaje que son analizados en el marco de un proceso de implementación en un aula de Ciencias, al tratarse de un análisis detallado y en profundidad solo fue posible analizar un caso, podría ser de utilidad el empleo de otro tipo de metodologías como el uso de rubricas, estudios longitudinales que permitan estudiar más casos y en consecuencia ampliar el volumen de información obtenida. En sintonía con lo anterior, el análisis en profundidad de un caso arroja un volumen elevado de información, por lo cual se sugiere para futuras investigaciones el empleo del software Atlas.Ti versión WIN 7.5.10 (Build 6) importante para el procesamiento de datos en investigaciones cualitativas.

Otra limitación tiene que ver con la granularidad de los OA, el tamaño puede limitar el proceso de implementación que la docente realice, una especie de cansancio o agotamiento generalizado puede ser causa de abandono. En ese sentido, reflexionar previamente acerca de la pertinencia del empleo de un recurso de un gran tamaño es pertinente. 


\section{REFERENCIAS BIBLIOGRÁFICAS}

Angeli, C., \& Valanides, N. (2009). Epistemological and methodological issues for the conceptualization, development, and assessment of ICT-TPCK: Advances in technological pedagogical content knowledge (TPCK). Computers \& Education, 52(1), 154-168. DOI: https://doi.org/10.1016/j.compedu.2008.07.006

Astudillo, G. J. (2011). Análisis del estado del arte de los objetos de aprendizaje. Revisión de su definición y sus posibilidades (Tesis de especialización). Universidad Nacional de La Plata, Buenos Aires, Argentina. Recuperado de https://core.ac.uk/download/pdf/15761403.pdf

Argento, D. (2013). Estudio exploratorio sobre preconcepciones en el área de Genética en alumnos de Secundaria italianos y españoles (Tesis de maestría). Universidad Internacional de la Rioja, Madrid, España.

https://reunir.unir.net/bitstream/handle/123456789/1425/2013_01_30_tfm_estudio_del_trabaj o.pdf?sequence=1\&isAllowed=y

Balatsoukas, P., Morris, A., \& Brien, A. (2008). Learning Objects Update: Review and Critical Approach to Content Aggregation. Educational Technology \& Society, 11(2), 119-130. Recuperado de https://www.researchgate.net/journal/Educational-Technology-Society-1436-4522

Blancas Hernández, J. L., \& Rodríguez Pineda, D. P. (2013). Uso De Tecnologías en la Enseñanza de las Ciencias. El Caso De Una Maestra De Biología De Secundaria. Revista Latinoamericana de Estudios Educativos, $\quad 9(1), \quad 162-186 . \quad$ Recuperado de https://www.redalyc.org/pdf/1341/134129372008.pdf

Callejas, M., Hernández, E. J., \& Pinzón, J. N. (2011). Objetos de aprendizaje, un estado del arte. Entramado, 7(1), 176-189. Recuperado de http://www.scielo.org.co/scielo.php?script=sci_arttext\&pid=S1900-38032011000100012

Carrillo, M. J., \& Roa, L. C. (2018). Diseñando el aprendizaje desde el modelo ADDIE. (Tesis de especialización) Universidad de La Sabana, Chía, Colombia. Recuperado de https://intellectum.unisabana.edu.co/handle/10818/35378

Carneiro, R. (2009). Las TIC y los nuevos paradigmas educativos: la transformación de la escuela en una sociedad que se transforma. En R. Carneiro, J. C. Toscano, \& T. Díaz (Coord.), Los desafíos de las TIC para el cambio educativo. Madrid: Fundación Santillana. (pp. 15-28). Recuperado de https://dialnet.unirioja.es/servlet/articulo?codigo $=5776496$ 
Castillo, M. C., \& Zambrano, A. C. (2020). Implementación de un objeto de aprendizaje: el caso de una docente de enseñanza secundaria.

Revista Convergencia Educativa 8, diciembre, 6-30. DOI: http://doi.org/10.29035/rce.8.6

Coll, C. (2008). Aprender y enseñar con las TIC. Expectativas, realidad y potencialidades. Boletín de la institución libre de enseñanza, 72(1), 17-40. Recuperado de https://dialnet.unirioja.es/servlet/articulo?codigo=3071971

Coll, C. (2004). Psicología de la educación y prácticas educativas mediadas por las tecnologías de la información y la comunicación. Una mirada constructivista. Sinéctica, Revista Electrónica de Educación, (25),1-24. Recuperado de https://www.redalyc.org/pdf/998/99815899016.pdf

Coll, C., Onrubia, J., \& Mauri, T. (2007). Tecnología y prácticas pedagógicas: las TIC como instrumentos de mediación de la actividad conjunta entre profesores y estudiantes. Anuario de Psicología, 38(3), 377-400. Recuperado de https://revistes.ub.edu/index.php/Anuariopsicologia/article/viewFile/8407/10382

Collazos, C., Pantoja, L., Hernández., U., Solarte, M., Agredo Mendez, G., \& Vásquez., G. (2007). Evaluando objetos de aprendizaje: Un caso práctico en la enseñanza de la electrónica. Revista Avances en Sistemas e Informática, 4(1), 137-144. Recuperado de https://repositorio.unal.edu.co/bitstream/handle/unal/24107/9726-16983-1PB.pdf?sequence $=1 \&$ isAllowed $=\mathrm{y}$

Chiappe Laverde, A. (2009). Objetos de aprendizaje 2.0: una vía alternativa para la re-producción colaborativa de contenido educativo abierto. En C. T. Valencia M., \& A. T. Jiménez H. (Ed.), Objetos de Aprendizaje. Prácticas y perspectivas educativas. (pp. 61-77). Santiago de Cali: Pontificia Universidad Javeriana. Recuperado de https://www.researchgate.net/publication/209388383_Objetos_de_Aprendizaje_20_una_via_alte rnativa_para_la_re-produccion_colaborativa_de_contenido_educativo_abierto

Díaz Barriga, F. (2008). Educación y nuevas tecnologías de la información: ¿Hacia un paradigma educativo innovador? Revista Electrónica Sinéctica, (30), 1-15. Recuperado de https://www.redalyc.org/pdf/998/99819167004.pdf

Díaz Quero, V. (2006). Formación Docente, Práctica Pedagógica y Saber Pedagógico, Revista de educación Laurus, 12(Ext.), 88-103. Recuperado de https://www.redalyc.org/pdf/761/76109906.pdf

Galvis, H. A., López, Y. Y., \& Aarón, M. A. (2018). Aprendiendo de una transformación de prácticas pedagógicas en cursos de maestría en la modalidad híbrida de aprendizaje. Revista de Educación a Distancia (RED), 18(58), 1-35. Recuperado de https://revistas.um.es/red/article/view/350951

García Aretio, Lorenzo (2005). Objetos de aprendizaje. Características y repositorios. Recuperado de http://e-spacio.uned.es/fez/eserv/bibliuned:327/editabril2005.pdf 
Castillo, M. C., \& Zambrano, A. C. (2020). Implementación de un objeto de aprendizaje: el caso de una docente de enseñanza secundaria.

Revista Convergencia Educativa 8, diciembre, 6-30. DOI: http://doi.org/10.29035/rce.8.6

González García, A., Gómez Zermeño, M. G., \& Abrego Tijerina, R. F. (2014). Implementación de un objeto de aprendizaje en un curso de formación docente. REXE Revista de Estudios y Experiencias en Educación, 13(26), 13-31. Recuperado de https://www.redalyc.org/comocitar.oa?id=243132847001

González Ruiz, L. M. (2016). Propuesta para evaluar la calidad de los objetos de aprendizaje mediante el uso de ontologías. (Tesis doctoral), Universidad de Alicante, Alicante, España. Recuperado de https://rua.ua.es/dspace/bitstream/10045/53886/1/tesis_gonzalez_ruiz.pdf

Harris, J., \& Hofer, M. (2009). Instructional Planning Activity Types as Vehicles for Curriculum-Based TPACK Development. En I. Gibson, R. Weber, K. McFerrin, R. Carlsen, \& D. Willis (Eds.), Proceedings of SITE 2009--Society for Information Technology \& Teacher Education International Conference. Conferencia llevado a cabo en Charleston, SC, Estados Unidos. Recuperado de https://www.learntechlib.org/p/31298/

Hsu, I-Ching (2017). Intelligent Discovery for Learning Objects Using Semantic Web Technologies. International Forum of Educational Technology \& Society. Journal of Educational Technology \& Society 15(1), 298-312. Recuperado de https://www.jstor.org/stable/jeductechsoci.15.1.298

Kay, R. H., \& Knaack, L. (2007). Evaluating the learning in learning objects. Open Learning, 22(1), 5-28. D0I: https://doi.org/10.1080/02680510601100135

Kay, R. H., \& Knaack, L. (2009). Analysing the Effectiveness of Learning Objects for Secondary School Science Classrooms. Journal of Educational Multimedia and Hypermedia, (18), 113-135. Recuperado de https://www.researchgate.net/publication/236134661_Analysing_the_Effectiveness_of_Learning _Objects_for_Secondary_School_Science_Classrooms

Ley No. 115. Diario Oficial de Colombia, Bogotá D.C., Colombia, 8 de febrero de 1994, Recuperado de http://www.alcaldiabogota.gov.co/sisjur/normas/Norma1.jsp?i=292

Ministerio de Educación Nacional (2011). Recursos educativos digitales abiertos. Estado del arte, Objetos de Aprendizaje. Contexto Nacional 2005-2011. Recuperado de http://aprende.colombiaaprende.edu.co/sites/default/files/naspublic/libroreda_0.pdf

Moreira, M. A. (2010). El proceso de integración y uso pedagógico de las TIC en los centros educativos, $\begin{array}{lllll}\text { Revista } & \text { Educación (352), 77-97. Recuperado de }\end{array}$ http://www.revistaeducacion.educacion.es/re352/re352_04.pdf

Naciones Unidas (2013). Objetivos de Desarrollo del Milenio. Nueva York: Naciones Unidas. Recuperado de http://mdgs.un.org/unsd/mdg/Resources/Static/Products/Progress2013/Spanish2013.pdf 
Polsani, P. R. (2003). Use and Abuse of Reusable Learning Objects. Journal of Digital Information, 3(4), 2-7. Recuperado

https://www.researchgate.net/publication/215439566 Use and Abuse of Reusable Learning 0 bjects

Wiley, D. A. (2002). Learning Objects Explained. En D. A. Wiley (Ed.), The Instructional Use of Learning Objects. Bloomington, Indiana: Agency for Instructional Technology Association for Educational $\begin{array}{lllll}\text { Communications } & \& & \text { Technology. } & \text { Recuperado }\end{array}$ https://members.aect.org/publications/InstructionalUseofLearningObjects.pdf

\section{Datos de correspondencia}

María Camila Castillo Cabezas

Magíster en Educación con énfasis en Enseñanza de las Ciencias

Universidad del Valle, Santiago de Cali, Colombia

ORCID ID: https://orcid.org/0000-0003-4086-9686

Email: maria.castillo.cabezas@correounivalle.edu.co 